\title{
Effects of C-peptide on the single-nephron glomerular filtration rate in early diabetic rats
}

\author{
Hiroshi Nakamoto ${ }^{1,2,3, *}$ \\ ${ }^{I}$ Nozaki Tokushukai Hospital Research Institute, Daito, Osaka, Japan \\ ${ }^{2}$ Department of Medical Engineering and Systems Cardiology, Kawasaki Medical School, Kurashiki, Okayama, Japan \\ ${ }^{3}$ Department of Clinical Engineering, Kawasaki Welfare University, Kurashiki, Okayama, Japan
}

Received: 30 October 2019 / Accepted: 6 May 2020

(C) Japanese Society of Biorheology 2020

\begin{abstract}
Objective: Single-nephron glomerular filtration rate (SNGFR) is an important and valuable parameter for evaluating renal function. Previously, SNGFR measurements required radioactive labelling of isolated animal nephrons, which was a time-consuming and inefficient process. Owing to the development of laser confocal microscopy, in vivo renal visualisation became much simpler, facilitated by the use of fluorescent markers. In this study, the effects of C-peptide on hyperfiltration reversal were evaluated during the early stages of diabetes mellitus (DM). Methods: Wistar rats $(n=17)$ were divided into control and streptozotocin-induced DM groups. Analyses were performed at 2 and 6 weeks following induction. Lucifer Yellow was administered by bolus injection to visualise tubular urinary flow using a laser confocal microscope. Results: SNGFR increased in diabetic rats $(p<0.05)$ and decreased by approximately $40 \%$ following C-peptide administration $(p<0.05)$. Moreover, tubular luminal flow increased $(p<0.05)$, as previously reported, but decreased by $3-11 \%$ after C-peptide administration in 6-week diabetic rats without significance. Conclusion: Confocal microscopy demonstrated that C-peptide administration decreases SNGFR and tubular luminal flow in diabetic rats.
\end{abstract}

Keywords diabetes, C-peptide, single-nephron glomerular filtration rate, hyperfiltration, hypertrophy Abbreviations used SNGFR, single-nephron glomerular
filtration rate; DM, diabetes mellitus; STZ, streptozotocin;
HbA1c, glycated haemoglobin A1c; FITC, fluorescein
isothiocyanate; BSA, bovine serum albumin; ANOVA,
analysis of variance

*E-mail: hershey@mx3.tiki.ne.jp

\section{Introduction}

Since the introduction of laser confocal microscopy in the late $20^{\text {th }}$ century, major advances in visualisation of renal microcirculation have been made [1-3]; one of these is that the nephroprotective effects of C-peptide can be detected by visualising glomerular hyperfiltration reversal in vivo, observed during the early stage of diabetes mellitus (DM) $[4,5]$. C-peptide was considered physiologically inactive; however, beneficial effects on early-stage DMinduced functional and structural abnormalities of the peripheral nerves, the kidneys, and the retina have been demonstrated [6-9]. Previously, determining the singlenephron glomerular filtration rate (SNGFR) from an isolated single nephron using radioactive markers required multiple days [10-12], Lorenz and Gruenstein developed a simple, nonradioactive method using fluorescein isothiocyanate (FITC)-inulin for evaluating SNGFR in 1999 [13]. Kang et al. succeeded in establishing a simpler in vivo measurement method for SNGFR in the mid-2000s using confocal microscopy [14]. Notably, SNGFR can be measured from video recordings under in vivo conditions.

Renal hypertrophy is an early manifestation of DM. Several growth factors are associated with renal hypertrophy including changes in tubular diameters $[15,16]$. The changes in tubular diameters in response to C-peptide have not been described in detail. Accordingly, in this study, the effects of C-peptide on hyperfiltration reversal were examined by measuring SNGFR instead of the relative sieving coefficient, as proposed in a previous study [4]. The effects of C-peptide and DM on changes in tubular diameter were also examined. 


\section{Material and Methods}

\section{Animals and surgical procedures}

Seventeen Wistar rats were used in this study. Type 1 DM was induced by tail-vein injection of streptozotocin (STZ, $50 \mathrm{mg} / \mathrm{kg}$ ) in saline. Control rats were injected with the same volume of saline. Rats were used in experiments 0,2 , and 6 weeks after injection of STZ or saline. For SNGFR visualisation, rats were continuously anaesthetised using inhaled sevoflurane (2\%; Maruishi Pharmaceutical, Osaka City, Japan) under spontaneous breathing after their body weights were measured. A cannula was then inserted into the tail vein for drug administration.

Glycated haemoglobin (HbAlc) levels were measured from a 1-mL blood sample by Special Reference Laboratories (SRL, Tokyo, Japan) using the latex coagulation method. The results confirmed that DM had been induced successfully because $\mathrm{HbAlc}$ reflects the average plasma glucose concentration over the preceding 4-12 weeks. The reference range for healthy humans is $4-5 \%$, according to the National Glycohemoglobin Standardization Program of the United States of America. The left kidney was exposed through a left flank incision. Observations were performed within the range of plausible measurement depths, at 10-100 $\mu \mathrm{m}$. The maximum accessible depth was more than 1,000 $\mu \mathrm{m}$ from the surface.

Rats were placed on a stage with the exposed left kidney facing the observation window and observed from underneath using an objective inverter (LSM Technologies Inc., Etter, PA, USA; Figure 1). The body of the rat held the left kidney in place from above, reducing movement of the kidney caused by respiration.

\section{Experimental protocol}

Rats were divided into three groups: nondiabetic with or without C-peptide $(\mathrm{n}=5), 2$-week diabetic rats with or without C-peptide $(\mathrm{n}=5)$, and 6-week diabetic rats with or without $\mathrm{C}$-peptide $(\mathrm{n}=7)$. C-peptide was continuously and intravenously administered at a dose of $50 \mathrm{pmol} / \mathrm{kg} / \mathrm{min}$ [17] and at a speed of $2 \mathrm{~mL} / \mathrm{h}, 60 \mathrm{~min}$ prior to the experiments [1] because its full effects are not observed until approximately $45 \mathrm{~min}$ after administration.

Next, $0.3 \mathrm{~mL}$ Lucifer Yellow solution (Thermo Fisher Scientific, MA USA; emission: $536 \mathrm{~nm}, 0.3 \mathrm{mg} / \mathrm{mL}$ ) was administered to visualise the primitive urinary flow. For example, approximately $90 \mathrm{~s}$ was required for each dose, with at least 3 min between doses. In most experiments, 12 sets were performed: six before and six after C-peptide administration. When pre-treatment with $\mathrm{C}$-peptide was performed, continuous administration of C-peptide was started $60 \mathrm{~min}$ prior to the experiment. The total length of the experiment, including surgical operation and C-peptide usage, was therefore nearly $3 \mathrm{~h}$. Because the dye solution was administered a dozen times, fluid compensation was not performed to avoid fluid overload.

\section{Measurement of SNGFR}

Analyses were performed after the experiments using video recordings. SNGFR was calculated as the tubular primitive urinary flow per minute. There was a time lag from the appearance of urinary flow between the upper and lower streams (Figure 2). Tubules were presumed to be cylindrical. SNGFR was calculated as $\pi R^{2} L$, where $R$ is the tubular radius and $L$ is the route of length between the upper and lower streams. The radius was the halved mean diameter measured at the upper, middle, and lower streams. From one rat, approximately 7 to 12 SNGFRs were obtained.

\section{Measurement of tubular diameters}

In an additional experiment, tubular diameters were measured in separate rats from the main experiment by changing the weeks and conditions $(n=5$ for each control, 2-week diabetic and 6-week diabetic groups). The diameters were measured from the tangential renal cortex in fixed sections stained with haematoxylin and eosin. Only round

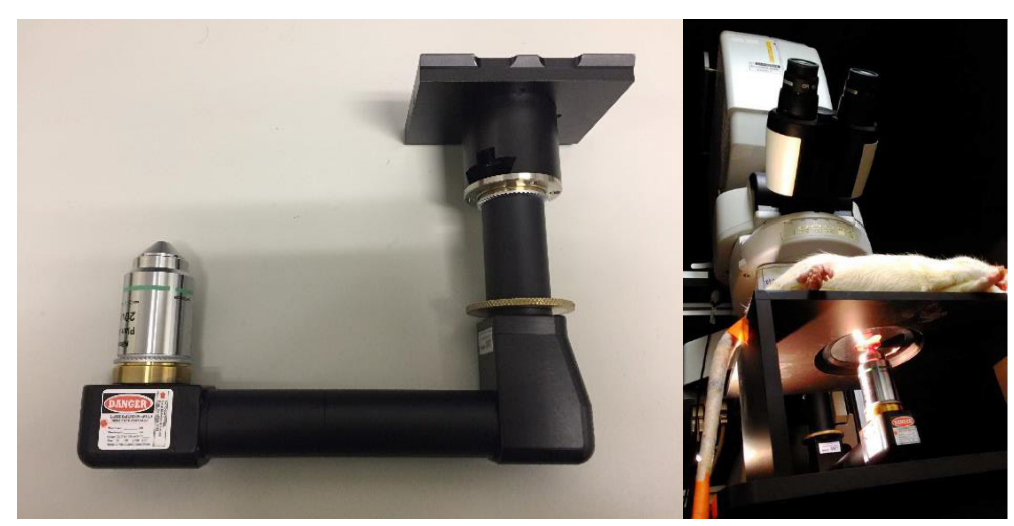

Figure 1 The objective inverter microscope InverterScope (LSM Technologies Inc., Etter, PA, USA). Renal microcirculation was observed from beneath using an objective inverter, converting an upright microscope into an inverted microscope. 


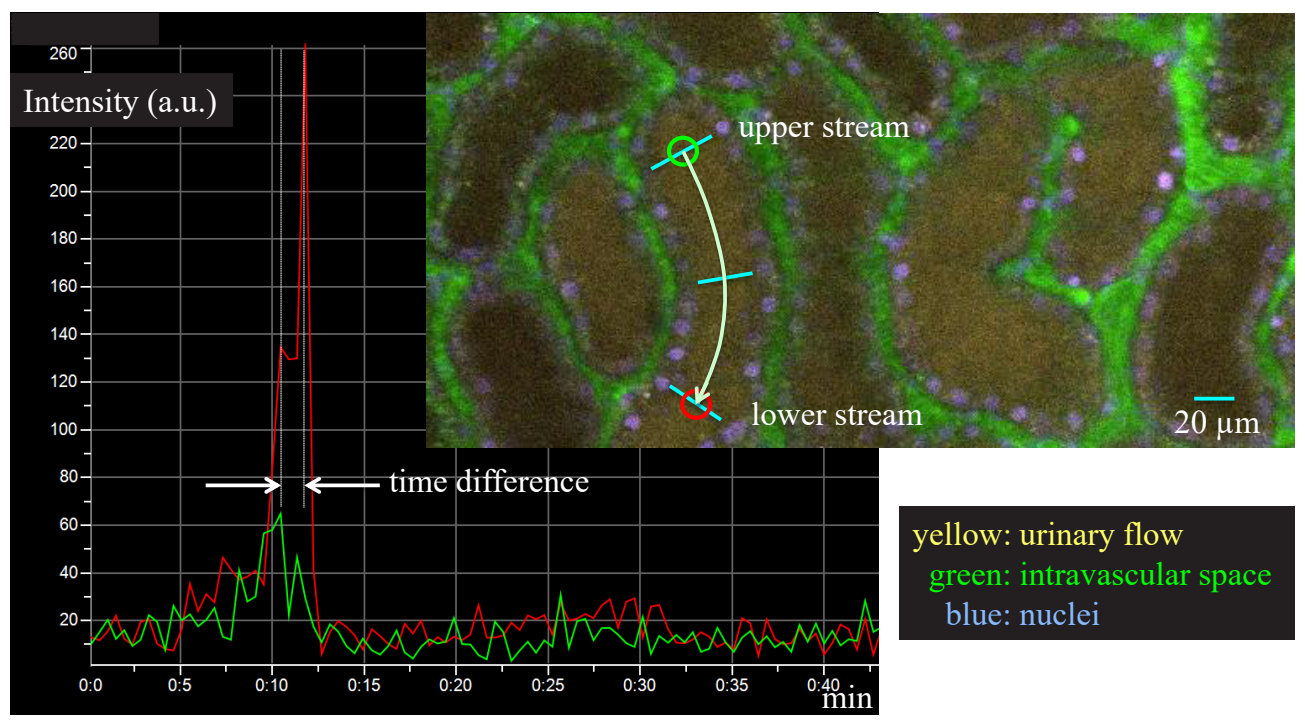

Figure 2 Tubular urinary flow visualisation and analysis. Tubular flow was visualised by a bolus of Lucifer Yellow. There was a time lag of the appearance of flow between the upper (green circle) and lower (red circle) streams. Assuming the tubules were cylindrical, the flow volume from the upper stream to the lower stream per minute could be calculated as SNGFR. Diameters were calculated as the mean values of diameters measured at the upper, middle, and lower streams. The length was the route from the upper to the lower stream.

tubules (minimum diameter/maximum diameter $\geq 0.8$ ) were analysed. Fifteen to thirty tubules were counted for each rat.

\section{Staining}

Bovine serum albumin conjugated to Alexa Fluor 594 (Thermo Fisher Scientific; emission: $617 \mathrm{~nm}$ ) was administered prior to the experiment to stain the plasma and intravascular space. Cell nuclei were stained using Hoechst 33342 (Invitrogen, Molecular Probes, Inc., Eugene, OR, USA; emission: $461 \mathrm{~nm}$ ) administered via the tail vein. For fixed sections, haematoxylin and eosin staining was performed.

\section{Multiphoton microscopy}

A multiphoton confocal laser microscope (A1R MP+; Nikon Corporation, Tokyo, Japan) was used to visualise renal microcirculation. After clearly capturing renal tubular microcirculation under in vivo conditions, Lucifer Yellow was administered $10 \mathrm{~s}$ after the beginning of the recording and for the next $80 \mathrm{~s}$ as tubular microcirculation was observed.

\section{Statistical analysis}

Data are expressed as means \pm standard errors. SNGFR and tubular diameters were assessed by analysis of variance (ANOVA). The effect of C-peptide was analysed by unpaired $t$-test. Differences with $p$ values less than 0.05 were considered statistically significant.

\section{Animal care}

All protocols were approved by the Committee on Animal Research of Kawasaki Medical School (approval nos. 15-33 and 17-22) and Nozaki Tokushukai Hospital Research Institute (approval no. 30-A-3-\#13). All animal care protocols and experiments complied with the Guidelines of the National Institutes of Health of the United States of America. The experimental procedures were performed in accordance with the guidelines of the approving institutions and those of the National Research Council of the National Academies of the United States of America.

\section{Results}

Body weights, blood sugar levels, and HBAlc levels

There were significant differences in body weight between the control and 2-week diabetic rats. Diabetes mellitus reduced body weights, although rats gradually regained weight so that there were no differences at 6 weeks (Table 1).

Table 1 Body weights, blood sugar levels, and $\mathrm{HbAlc}$ values

\begin{tabular}{cccc}
\hline & Control & 2-weeks DM & 6-weeks DM \\
\hline $\begin{array}{c}\text { Number of rats } \\
\text { Body weight }(\mathrm{g})\end{array}$ & 5 & 5 & 7 \\
$\begin{array}{c}\text { Blood sugar levels } \\
(\mathrm{mg} / \mathrm{dL})\end{array}$ & $140.6 \pm 5.3$ & $340.6 \pm 33.6^{*}$ & $422.3 \pm 23.2^{*}$ \\
HBA1c $(\%)$ & $5.1 \pm 0.1$ & $8.5 \pm 0.2^{*}$ & $10.4 \pm 0.1^{*}$ \\
\hline
\end{tabular}

Data are means \pm SEMs. ${ }^{*} p<0.05$ 
Measurement of other parameters revealed significant differences in blood sugar levels between control rats and diabetic rats (Table 1). Moreover, significant differences in HbA1c were observed between control and diabetic rats, confirming that the control rats were not diabetic (Table 1).

\section{SNGFRs and tubular diameters}

There were significant differences in SNGFR between control and diabetic groups. SNGFRs were $6.3 \pm 1.5,11.6 \pm$ 1.4 , and $15.2 \pm 3.1 \mathrm{~nL} / \mathrm{min}$ for control, 2-week diabetic, and 6-week diabetic rats, respectively. DM increased SNGFR in a time-dependent manner, with greater increases observed after longer times. Notably, C-peptide administration reduced SNGFR only in 6-week diabetic rats. SNGFRs after C-peptide administration were $5.2 \pm 1.8,8.6 \pm 3.2$ and $10.7 \pm 2.3 \mathrm{~nL} / \mathrm{min}$ for control, 2-week diabetic, and 6-week diabetic rats, respectively (Figure 3).

Tubular luminal flow was clearly visualized over 90 seconds. From off-line video measurements, significant differences were observed in tubular diameters. Tubular diameters were $20.1 \pm 0.3,29.3 \pm 2.1$, and $30.1 \pm 1.1 \mu \mathrm{m}$ for control, 2-week diabetic, and 6-week diabetic rats, respectively. DM-induced increases in tubular diameters may be dependent on the duration of diabetes, with larger increases observed after longer disease durations. Tubular diameters after C-peptide administration were $20.0 \pm 0.5$, $28.3 \pm 1.9$, and $26.7 \pm 1.5 \mu \mathrm{m}$ for control, 2-week diabetic, and 6-week diabetic rats, respectively. Thus, C-peptide did not decrease diameters in all groups (Figure 4).

In an additional experiment based on analysis of fixed renal tissue samples, significant differences in tubular diameters between control and diabetic rats without C-peptide administration were observed $(21.0 \pm 1.0,25.8 \pm 1.4$, and

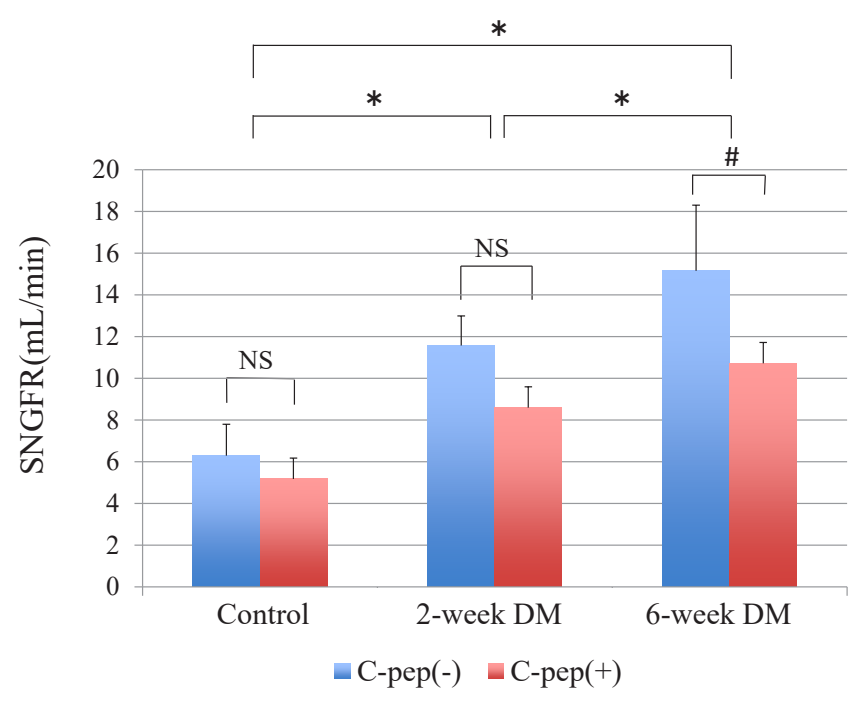

Figure 3 Effects of C-peptide on SNGFR. Differences between groups were analysed by ANOVA $\left({ }^{*} p<0.05\right)$. C-peptide effects were analysed by unpaired $t$-test $\left({ }^{\#} p<0.05\right)$. NS, not significant.
$28.5 \pm 2.2 \mu \mathrm{m}$ for control, 2-week diabetic, and 6-week diabetic rats, respectively, $p<0.05)$. These findings confirmed that DM increased tubular diameters, but there was not a significant difference between 2- and 6-week diabetic rats (Figures 5 and 6).

\section{Discussion}

This study demonstrated that C-peptide reversed diabetic renal hyperfiltration using SNGFR as an index for the first time. An objective inverter, which converts an upright microscope into an inverted microscope, was employed to visualise renal tubular flow with minimised movement due to respiration. Moreover, tubular luminal diameter increased because of DM, as previously reported [14], but decreased after C-peptide administration, which was not statistically significant and has not been reported in prior studies.

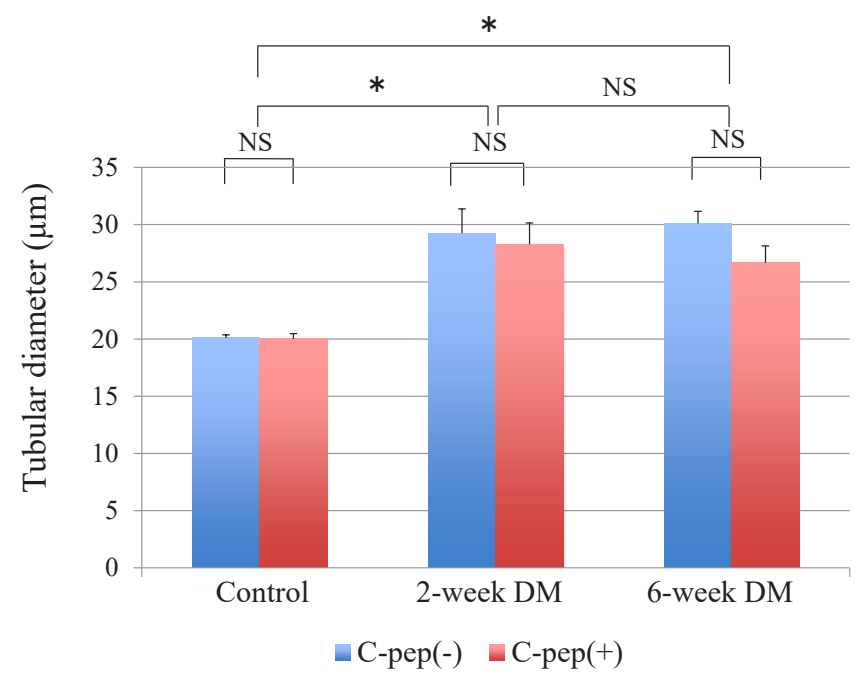

Figure 4 Differences in tubular diameters between control and diabetic rats treated with or without C-peptide. Differences between groups were analysed by ANOVA $(* p<0.05)$. C-peptide effect was analysed by unpaired $t$-test $\left({ }^{\#} p<0.05\right)$. NS, not significant.

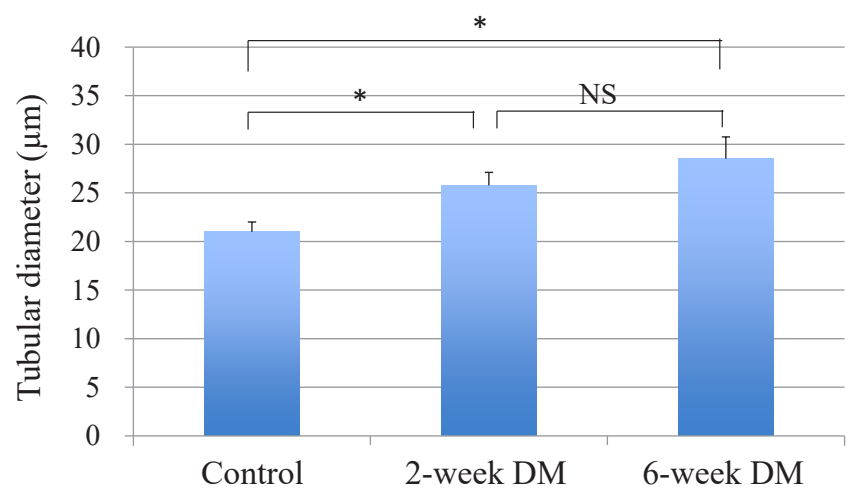

Figure 5 Differences in tubular diameters between control and diabetic rats. Differences between groups were analysed by ANOVA $(* p<0.05)$. NS, not significant. 


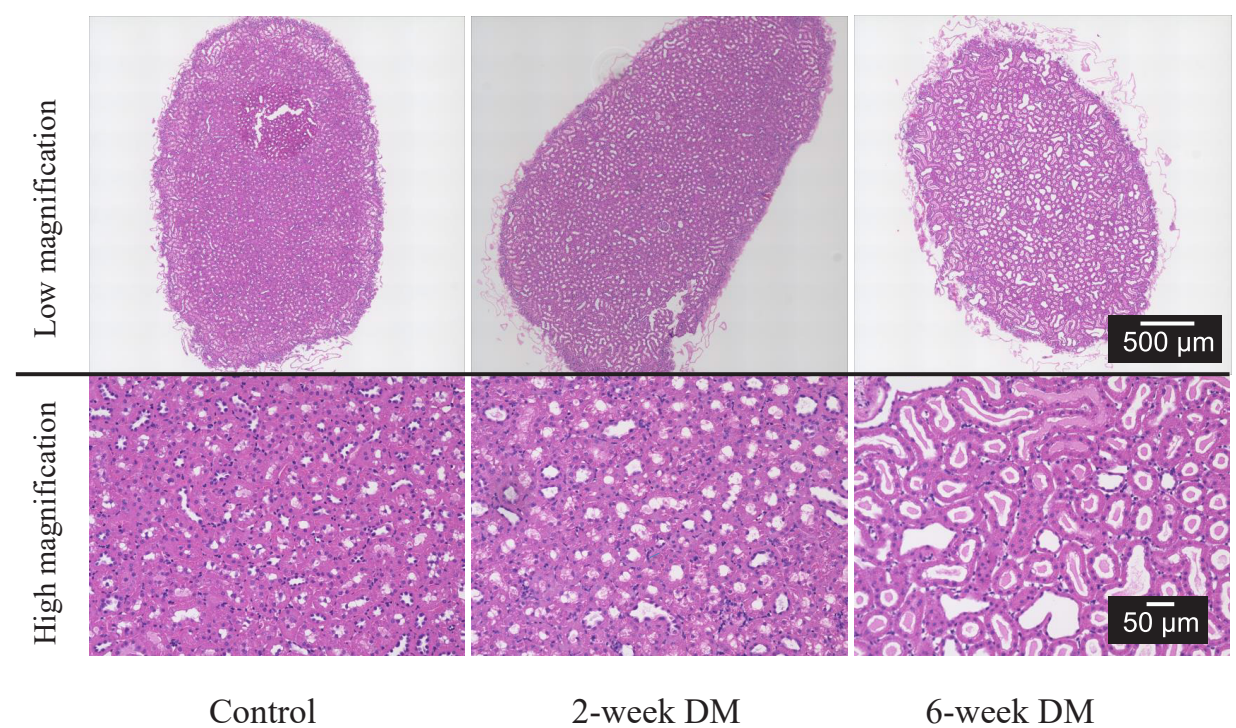

Figure 6 Representative fixed sections of renal tissues stained with haematoxylin and eosin. Diameters were analysed only for the round tubules (minimum diameter/maximum diameter $\geq 0.8$ ) sectioned along the short axis. Upper panels show images at low magnification, and lower panels show images of high magnification.

Diabetic nephropathy is initially characterized by hyperfiltration. Several studies have shown that acute and longterm infusion of C-peptide reduces the hallmarks of early diabetic nephropathy, namely glomerular hyperfiltration and glomerular hypertrophy [9]. Notably, C-peptide reduced hyperfiltration, using the relative sieving coefficient as an index [4]. C-peptide constricts glomerular afferent arterioles and dilates efferent arterioles [18, 19]. Thus, when C-peptide is administered, glomerular filtration pressure and rate are reduced resulting in reduced SNGFR [4].

Measuring SNGFR has been difficult. Initial approaches for measuring it required micropuncturing several sites of a nephron and collecting fluid from the tubules of the same nephron, a technically difficult task. However, SNGFR can now be measured easily using confocal microscopy, as shown by Kang et al. [14]. Under in vivo conditions, Lucifer Yellow is intravenously administered and SNGFR can be measured from video images of tubular flow after the experiment.

Kang et al. demonstrated differences in SNGFR between control and diabetic rats [14], consistent with our results. However, our findings demonstrated the effects of C-peptide on reversal of diabetic renal hyperfiltration for the first time. In previous reports, this C-peptide effect was observed using an index called the relative sieving coefficient, which represents glomerular filtration function in an in vivo, dynamic state. Because SNGFR is directly connected to glomerular filtration rate, it is a more direct measurement of renal function than the relative sieving coefficient.

The SNGFRs obtained in this study were lower than those previously reported $[11,14]$. SNGFRs are affected by age, sex, body weight, kidney weight, season, and other factors. Some of these factors, or the method used to measure diameters, may be responsible for this difference. In this study, tubules with lengths of more than $80 \mu \mathrm{m}$, slightly shorter than those reported by Kang et al. [14], were used. In any case, the qualitative relationships expected for DM and C-peptide with SNGFR were demonstrated in the new dataset presented in this study.

There was a tendency for tubular diameter to increase with the duration of DM and there was no significant DMinduced increase in tubular diameters between 2-week diabetic and 6-week diabetic rats (Figures 4-6). The reason for these results may be owing to the slight displacing movement due to respiration inevitable for in-vivo observation by confocal microscopy. Larger sample sizes would likely reveal a significant difference between the 2-week and 6-week diabetic rats. It is because transforming growth factor- $\beta$ induced by hyperglycaemia is reponsible for hypertrophy of tubular cells [20].

Tubules with lengths greater than $80 \mu \mathrm{m}$ were chosen for SNGFR analyses. Visual inspection of these revealed luminal flow was homogenous even in the tubules where SNGFR was not measured. Glomerular filtration is typically thought to be heterogeneous. Although we did not quantitatively examine the heterogeneity of tubular flows, the tubules observed by video image showed transient homogeneous luminal flow after dye solution administration.

Diabetes mellitus causes renal hypertrophy. During the process of measuring SNGFRs, the current findings revealed enlargement of tubular diameters caused by DM, as was reported decades ago [21]. An additional experiment with fixed renal sections supported this finding. Theoretically, the direct comparison of diameters between in-vivo conditions and fixed samples cannot be done, because fixed samples are said to be shrunk by 5-10\%. Only the qualitative changes caused by DM have significance. In reality, 
there was not much difference as to the diameters between in-vivo observation and fixed sample measurements.

Renal hypertrophy related to DM is caused by several growth factors, which also affect the tubular structures. Epidermal growth factor and insulin-like growth factor-I have been shown to act in concert to promote renal growth in DM $[16,22]$. C-peptide partially reversed this enlargement in diabetic rats, without statistical significance. This effect may not be structural, but instead may be passive and related to reduced hyperfiltration and urinary flow. This is likely the reason why I did not observe significant flow reduction after C-peptide administration. The current study confirmed previous findings demonstrating an increase in tubular luminal diameter using multiphoton microscopy. Although there was not a significant difference in diameters after C-peptide administration, I did observe a statistically insignificant reduction.

In this study, C-peptide treatment had short-term effects on SNGFRs. Because the effects of C-peptide were studied for approximately $2 \mathrm{~h}$ after beginning continuous administration, they were considered to be non-structural. Although these findings supported a nephroprotective effect of C-peptide, they also suggest that C-peptide replacement prevents hyperfiltration during early-stage DM [23].

Reduced glomerular filtration rate after C-peptide administration results in reduced SNGFR. Urinary tubules undertake reabsorption and secretion of various substances such as proteins, glucose, urea, creatinine and electrolytes. In general, tubular reabsorption is quantitatively more important than tubular secretion in the formation of urine. Tubular cells consume energy during reabsorption or secretion processes by active transport of sodium, glucose etc. and by protein pinocytosis, especially during hyperfiltration, because there is more urinary flow than usual [24]. Reduced hyperfiltration means reduced tubular reabsorption and secretion, and energy consumption is also reduced at tubular cells.

If C-peptide is administered continuously, then the flowinduced glomerular enlargement is suppressed because hyperfiltration does not occur. Since the leading cause for diabetic glomerular hypertrophy is transforming growth factor induced by hyperglycaemia [20], C-peptide cannot suppress diabetic glomerular hypertrophy.

Urinary bladder works as a urinary storehouse. A tubule is a part of a nephron. A nephron is considered to work as a finely tuned safety valve, adjusting the urine volume and intravascular volume, because about $99 \%$ of urine is reabsorbed at the tubules and collecting ducts. Changes in glomerular filtration and tubular reabsorption usually act in a coordinated manner to produce the necessary changes in renal excretion. Otherwise, just a $10 \%$ increase in the glomerular filtration rate would increase the urine volume 13-fold if tubular reabsorption remained constant [24]. In this regard, tubular function is inextricably involved in the process of urine volume adjustment.

Short-term C-peptide administration reduced the glomerular filtration rate and protein leakage from the glomeruli, as reported in our previous study [4]; this is nephroprotective and perhaps contributes to plasma osmolarity sustainment for systemic circulation.

There were some limitations to this study. Glomerular filtration rates were not measured simultaneously. In a separate experiment, however, the presence of diabetic hyperfiltration was observed. Glomerular filtration rate was significantly higher in diabetic rats than in control rats in the absence of C-peptide. Our data demonstrated that the SNGFR increased in diabetic rats. Future studies are required to perform clinical analyses of C-peptide replacement, such as the clinical trials carried out by Wahren's group $[23,25]$. Additionally, further investigations of the molecular mechanisms of C-peptide effects are needed, with more detail than previous reports $[8,26]$.

\section{Conclusion}

This study demonstrated the nephroprotective hyperfiltration reversal effects of C-peptide for the first time using SNGFR as an index. Moreover, in vivo observation by confocal laser microscopy revealed a statistically insignificant tubular diameter decrease following C-peptide administration.

Acknowledgements Part of this work was presented at the annual meeting of the Japanese Circulation Society in 2018. For data collection, I wish to acknowledge the assistance of students in the Department of Clinical Engineering at Kawasaki Welfare University: T. Kojima, K. Murao, H. Moriue, A. Maeda, T. Umeji, T. Watanabe, M. Harada, M. Namba, A. Yoshikawa, J. Nakatsuji, M. Fukui, and K. Takase. This work was supported by a Grant-in-Aid from the Japanese Ministry of Education, Culture, Sports, Science, and Technology JSPS KAKENHI. JP15H03012 (B), 2015-2017, and a grant from Kawasaki Welfare University (2018).

\section{References}

1. Nakamoto H. Visualisation studies and glomerular filtration in early diabetic rats. J Biomech. 2017; 50: 138-43.

2. Peti-Peterdi J, Kidokoro K, Riquier-Brison A. Novel in vivo techniques to visualise kidney anatomy and function. Kidney Int. 2015; 88: 44-51.

3. Sipos A, Toma I, Kang JJ, Rosivall L, Peti-Peterdi J. Advances in renal (patho)physiology using multiphoton microscopy. Kidney Int. 2007; 72: 1188-91.

4. Nakamoto H, Kajiya F. In vivo quantitative visualisation analysis of the effect of C-peptide on glomerular hyperfiltration in diabetic rats by using multiphoton microscopy. Microcirculation. 2013; 20: 425-33.

5. Tanner GA. Glomerular sieving coefficient of serum albumin in the rat: a two-photon microscopy study. Am J Physiol Renal Physiol. 2009; 296: F1258-65.

6. Ido Y, Vindigni A, Chang K, Stramm L, Chance R, Heath WF, DiMarchi RD, Di Cera E, Williamson JR. Prevention of vascular and neural dysfunction in diabetic rats by C-peptide. Science. 1997; 277: 563-6.

7. Wahren J, Johansson BL, Wallberg-Henriksson H, Linde B, 
Fernqvist-Forbes E, Zierath JR. C-peptide revisited—new physiological effects and therapeutic implications. J Intern Med. 1996; 240: 115-24.

8. Wahren J, Larsson C. C-peptide: new findings and therapeutic possibilities. Diabetes Res Clin Pract. 2015; 107: 309-19.

9. Yosten GL, Maric-Bilkan C, Luppi P, Wahren J. Physiological effects and therapeutic potential of proinsulin C-peptide. Am J Physiol Endocrinol Metab. 2014; 307: E955-68.

10. Aukland K, Odd E. Hanssen and the Hanssen method for measurement of single-nephron glomerular filtration rate. Am J Physiol Renal Physiol. 2001; 281: F407-13.

11. Vallon V. Micropuncturing the nephron. Pflugers Arch. 2009; 458: 189-201.

12. Wright FS, Giebisch G. Glomerular filtration in single nephrons. Kidney Int. 1972; 1: 201-9.

13. Lorenz JN, Gruenstein E. A simple, nonradioactive method for evaluating single-nephron filtration rate using FITC-inulin. Am J Physiol. 1999; 276: F172-7.

14. Kang JJ, Toma I, Sipos A, McCulloch F, Peti-Peterdi J. Quantitative imaging of basic functions in renal (patho)physiology. Am J Physiol Renal Physiol. 2006; 291: F495-502.

15. Chiarelli F, Gaspari S, Marcovecchio ML. Role of growth factors in diabetic kidney disease. Horm Metab Res. 2009; 41: 585-93.

16. Sugimoto H, Shikata K, Makino H, Ota K, Ota Z. Increased gene expression of insulin-like growth factor-i receptor in experimental diabetic rat glomeruli. Nephron. 1996; 72: 648-53.

17. Forst T, Kunt T, Pohlmann T, Goitom K, Engelbach M, Beyer J, Pfützner A. Biological activity of C-peptide on the skin microcirculation in patients with insulin-dependent diabetes mellitus. J Clin Invest. 1998; 101: 2036-41.
18. Nordquist L, Lai EY, Sjöquist M, Patzak A, Persson AE. Proinsulin C-peptide constricts glomerular afferent arterioles in diabetic mice. A potential renoprotective mechanism. Am J Physiol Regul Integr Comp Physiol. 2008; 294: R836-41.

19. Nordquist L, Brown R, Fasching A, Persson P, Palm F. Proinsulin C-peptide reduces diabetes-induced glomerular hyperfiltration via efferent arteriole dilation and inhibition of tubular sodium reabsorption. Am J Physiol Renal Physiol. 2009; 297: F1265-72.

20. Wolf G, Ziyadeh FN. Molecular mechanisms of diabetic renal hypertrophy. Kidney Int. 1999; 56(2): 393-405.

21. Seyer-Hansen K, Hansen J, Gundersen HJ. Renal hypertrophy in experimental diabetes. A morphometric study. Diabetologia. 1980; 18: 501-5.

22. Gilbert RE, Cox A, McNally PG, Wu LL, Dziadek M, Cooper ME, Jerums G. Increased epidermal growth factor in experimental diabetes related kidney growth in rats. Diabetologia. 1997; 40: 778-85.

23. Wahren J, Kallas A, Sima AA. The clinical potential of C-peptide replacement in type 1 diabetes. Diabetes. 2012; 61: 761-72.

24. Hall JE. Guyton and Hall Textbook of Medical Physiology, Thirteenth Edition, International Edition. W B Saunders Co Ltd.; 2015. pp. 332, 347-50.

25. Wahren J, Foyt H, Daniels M, Arezzo JC. Long-acting C-peptide and neuropathy in type 1 diabetes: a 12 -month clinical trial. Diabetes Care. 2016; 39: 596-602.

26. Nakamoto H, Nakayama K, Emoto N, Kajiya F. The short-term effects of C-peptide on the early diabetes-related ultrastructural changes to the podocyte slit diaphragm of glomeruli in rats. Microcirculation. 2015; 22: 122-32. 